06,07

\title{
Релаксация поляризации при статическом прямом флексоэлектрическом эффекте в монокристалле $\mathrm{SrTiO}_{3}$
}

\author{
() В.Г. Залесский, Е.Д. Обозова, А.Д. Полушина, П.П. Сырников \\ Физико-технический институт им. А.Ф. Иофрфе РАН, \\ Санкт-Петербург, Россия \\ E-mail: nsh@mail.ioffe.ru
}

Поступила в Редакцию 16 мая 2021 г.

В окончательной редакции 16 мая 2021 г.

Принята к публикации 31 мая 2021 г.

Представлены результаты исследования диэлектрической поляризации, индуцированной сферическим изгибом тонких монокристаллических пластин $\mathrm{SrTiO}_{3}$, и произведена оценка флексоэлектрических коэффициентов. Исследованы процессы релаксации неоднородной деформации и индуцированной поляризации.

Ключевые слова: флексоэлектрический эффект, поляризация, механические свойства, релаксация, титанат стронция.

DOI: $10.21883 /$ FTT.2021.10.51406.116

\section{1. Введение}

Взаимосвязь между неоднородной деформацией и поляризацией диэлектрической среды получила название флексоэлектрического эффекта. Этот электромеханический эффект малозаметен в кристаллах макроскопического размера, однако в объектах микронного и субмикронного масштаба, например, в тонких пленках, он становится существенным, внося заметный вклад в ее поляризацию и деформацию. Поэтому эффект представляет интерес для практического применения в области интегральной электроники и в разработке микроэлектромеханических систем. Для описания связи между поляризацией и градиентом деформации используются флексоэлектрические коэффициенты, тензоры четвертого ранга $[1,2]$ :

$$
P_{i}=\mu_{k l i j} \frac{\partial u_{k l}}{\partial x_{j}}
$$

Порядок величины флексоэлектрических коэффициентов можно оценить по следующей формуле [1,2]:

$$
\mu \sim \chi \frac{e}{a},
$$

где $\chi-$ диэлектрическая восприимчивость, $e-$ заряд электрона, $a$ - параметр кристаллической ячейки. Поскольку в формуле (2) фигурирует диэлектрическая восприимчивость, то для исследования эффекта наиболее интересными объектами являются диэлектрики с большой диэлектрической проницаемостью, к которым относятся сегнетоэлектрики и родственные материалы. Для многих известных активных диэлектриков уже были проведены расчеты флексоэлектрических коэффициентов $a b$ inition др. методами [2]. В большинстве случаев, за исключением кристаллов $\mathrm{SrTiO}_{3}$, между теоретическими и экспериментально измеренными величинами наблюдается большое расхождение вплоть до нескольких порядков [2,3].
Виртуальный сегнетоэлектрик $\mathrm{SrTiO}_{3}$ (далее $\mathrm{ST}$ ) pacсматривается как модельный флексоэлектрический материал, поскольку обладает большой поляризуемостью $\left(\chi=300\right.$ при $\left.20^{\circ} \mathrm{C}\right)$, а центросимметричная кубическая фаза $P m \overline{3} m$ в широком температурном диапазоне не маскирует флексоэлектрический эффект пьезоэлектричеством. Кроме того, кристаллическая структура перовскита относительно проста для теоретических расчетов. Опубликованные в [4] теоретические расчеты давали следующие величины продольного и поперечного тензора $\mu_{11}=-0.264$ и $\mu_{12}=-3.75 \mathrm{nC} / \mathrm{m}$. В работе [5] по исследованию прямого флексоэлектрического эффекта в ST, было получено соответствие с точностью до порядка величины расчетных и экспериментально измеренных тензоров $\mu_{11}=0.2$ и $\mu_{12}=7 \mathrm{nC} / \mathrm{m}$. В другой работе [6] методом обратного эффекта был измерен эффективный тензор $\widetilde{\mu}_{12}=4.6 \mathrm{nC} / \mathrm{m}$. Можно отметить, что в этих двух работах для измерения тензора $\mu_{12}$ использовались переменные по времени внешние механические и электрические поля. Однако в экспериментах, где применялись статические или квазистатические поля, коэффициенты превышали расчетные на несколько порядков. Например, в работе [7] эффективный поперечный флексоэлектрический тензор $\widetilde{\mu}_{12}$ достигал величины $3.7-4.6 \mu \mathrm{C} / \mathrm{m}$ при исследовании обратного эффекта с использованием длительных импульсов высокого напряжения. В другой публикации [8] тот же тензор, измеренный по изгибу кристаллической поверхности около планарных электродов под высоким напряжением, был еще на три порядка больше и оценивался величиной $\widetilde{\mu}_{12}=6.334 \mathrm{mC} / \mathrm{m}$.

Согласно авторам статьи [4], причиной расхождения теоретических и экспериментальных оценок может быть чрезвычайно большая чувствительность поведения мягкой оптической моды к температуре и др. внешним воздействиям в сегнетоэлектрических перовскитах, которая, в свою очередь, должна сказываться на величине 
флексоэлектрических коэффициентов. При этом расчеты коэффициентов по динамике решетки предполагают нулевую температуру. Согласно этим расчетам для титанатов бария и титаната стронция флексоэлектрические коэффициенты имеют один и тот же порядок величины. Тем не менее, оба кристалла различаются диэлектрической восприимчивостью в сотни раз, что предполагает большую разницу и в величине флексоэлектрического отклика.

Другим подходом для оценки величин флексоэлектрических коэффициентов является введение в формулу (2) эмпирического размерного фактора $\lambda$, который зависит от геометрии образца (отношения толщины и длины образца), упругих свойств, фазового состава и технологии изготовления [1,2,9-11]:

$$
\mu \sim \lambda \chi \frac{e}{a}
$$

Для коэффициентов, рассчитанных в работе [4], величина $\lambda$ оценивается порядком $10^{-2}$, в то время как экспериментальные работы по титанату бария и соединений на его основе для поперечного флексоэлектрического тензора $\mu_{12}$ дают величины $\lambda \sim 1-10$ [10,11].

Расхождение в оценках флексоэлектрического коэффициента также может быть связано с вкладами различных ионных и электронных механизмов. Со стороны высоких частот возможен вклад собственных механических резонансов в образце, как это было показано в [6]. При низких частотах должны проявляться релаксационные и гистерезисные явления, например, за счет транспорта и перераспределения носителей заряда (электронов, ионов или кислородных вакансий) в объеме кристалла [12], а также за счет механической пластичности образца, которая, в свою очередь, обусловлена движением дислокаций и их взаимодействием с точечными дефектами [13].

В наших предыдущих работах сообщалось о наблюдении релаксационных и гистерезисных явлений, которые были причиной ряда экспериментальных сложностей при исследовании статического обратного флексоэлектрического эффекта в тонких монокристаллических пластинах ST [14]. В настоящей работе предлагается детальное исследование релаксационных процессов и оценка флексоэлектрических тензоров в условиях статического прямого эффекта. При этом предполагается использование деформации только сферического изгиба, что упрощает сравнение полученных данных с результатами исследования деформации того же типа при обратном эффекте [14]. Результаты сравнения могут дать дополнительную информацию о симметрии прямого и обратного флексоэлектрического эффекта.

\section{2. Эксперимент}

Образцами для исследования служили высококачественные монокристаллы ST, выращенные в ФТИ им. А.Ф. Иоффе методом Чохральского. После определения кристаллографических направлений рентгеновским

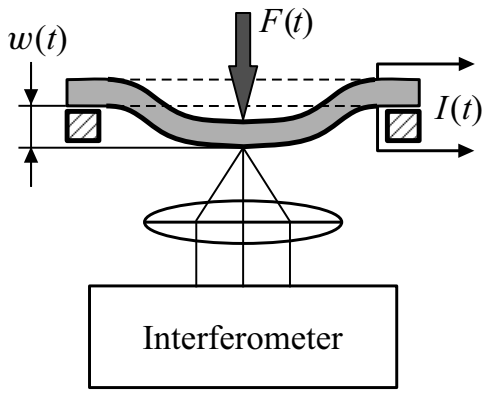

Рис. 1. Схема установка для исследования прямого флексоэлектрического эффекта.

дифрактометром ДРОН-3 монокристаллы были вырезаны в виде пластин толщиной $140 \mu \mathrm{m}$ с наибольшей поверхностью типа (001), площадь которой $11 \times 11 \mathrm{~mm}^{2}$. На отполированную до оптического качества поверхность методом термического напыления наносились золотые электроды диаметром $8 \mathrm{~mm}$.

Пластины были закреплены по краям к неподвижному круговому контуру с диаметром $10 \mathrm{~mm}$. Сферический изгиб производился механической нагрузкой с помощью иглообразного зонда из сапфира с острием диаметром $0.3 \mathrm{~mm}$, направленным в центр рабочей поверхности пластины (рис. 1). Электромеханический привод зонда обеспечивал периодическую механическую нагрузку $F$ в диапазоне от 0 до $1.5 \mathrm{~N}$ и длительностью от $1 \mathrm{~s}$ до десятков минут. Нагрузка $2 \mathrm{~N}$ была предельной и приводила к раскалыванию пластин. Скорость сближения или удаления острия от поверхности образца составляла $0.6 \mathrm{~cm} / \mathrm{s}$. В результате формировались импульсы механического напряжения $F(t)$ прямоугольной формы с периодом 5-6s и длительностью переднего и заднего фронта не больше $5 \cdot 10^{-4} \mathrm{~s}$.

Величина прогиба пластин $w$ измерялась микроскопом-интерферометром с разрешением до $10 \mathrm{~nm}$ [15]. Установка также позволяла отслеживать изменения в деформации за минимальный промежуток времени $0.05 \mathrm{~s}$. Индуцированный неоднородной деформацией ток поляризации измерялся с помощью электрометрического усилителя у5-11 с чувствительностью $10^{-15} \mathrm{~A}$ и временным разрешением не меньше $0.05 \mathrm{~s}$. Интегрирование плотности тока дает величину индуцированной поляризации.

\section{3. Результаты}

\section{1. Механические свойства}

Для исследования прямого флексоэлектрического эффекта необходимы предварительные измерения упругих и пластических свойств кристаллических пластин. Нагрузка иглообразным механическим зондом в диапазоне $F=0-1.5 \mathrm{~N}$ вызывала сферический изгиб с величиной прогиба центра пластины $w$ от 0 до $3.0 \mu \mathrm{m}$. Проверка 
соответствия экспериментальных данных с расчетными проводилась с помощью формулы сферического изгиба из теории деформации тонких пластин [16]:

$$
w=\frac{q r^{4}}{64 D}
$$

где: $r$ - радиус поверхности, свободной для изгиба, $q=F / S$ - механическое напряжение $\left(S=\pi r^{2}-\right.$ площадь поверхности), $D=\frac{h^{3} E_{Y}}{12\left(1-v^{2}\right)}-$ упругость на изгиб, $h$ - толщина кристаллической пластины, $E_{Y}-$ модуль Юнга, v - коэффициент Пуассона. Для расчетов использовались приведенные в предыдущем параграфе данные по размерам пластин, а параметры упругости $E_{Y}=228 \mathrm{GPa}$ и $v=0.237$ взяты из базы данных [17]. В частности, расчет прогиба по формуле (4) для механической нагрузки $F=0.5 \mathrm{~N}$ давал величину $w=0.9 \mu \mathrm{m}$. Этот расчет хорошо согласуется с экспериментом, в котором нагрузка той же величины обеспечивала максимальный прогиб $w_{\max }=1.05 \pm 0.50 \mu \mathrm{m}$ (рис. 2).

После снятия нагрузки наблюдалась релаксация деформации, сначала быстрым темпом, а затем более медленным. Временная зависимость величины прогиба $w(T)$, построенная в полулогарифмическом масштабе (рис. 2), позволила по линейным участкам выделить три процесса релаксации, которые можно описать следующим выражением:

$$
\begin{aligned}
w= & w_{01} \exp \left(-t / \tau_{1}^{\text {relax }}\right)+w_{02} \exp \left(-t / \tau_{2}^{\text {relax }}\right) \\
& +w_{03} \exp \left(-t / \tau_{3}^{\text {relax }}\right)
\end{aligned}
$$

где $w_{01}, w_{02}$, и $w_{03}-$ величины вкладов каждого процесса, а $\tau_{1}^{\text {relax }}, \tau_{2}^{\text {relax }}$ и $\tau_{3}^{\text {relax }}-$ соответствующие характерные времена, равные $1.3,6.5$ и $12.5 \mathrm{~s}$.

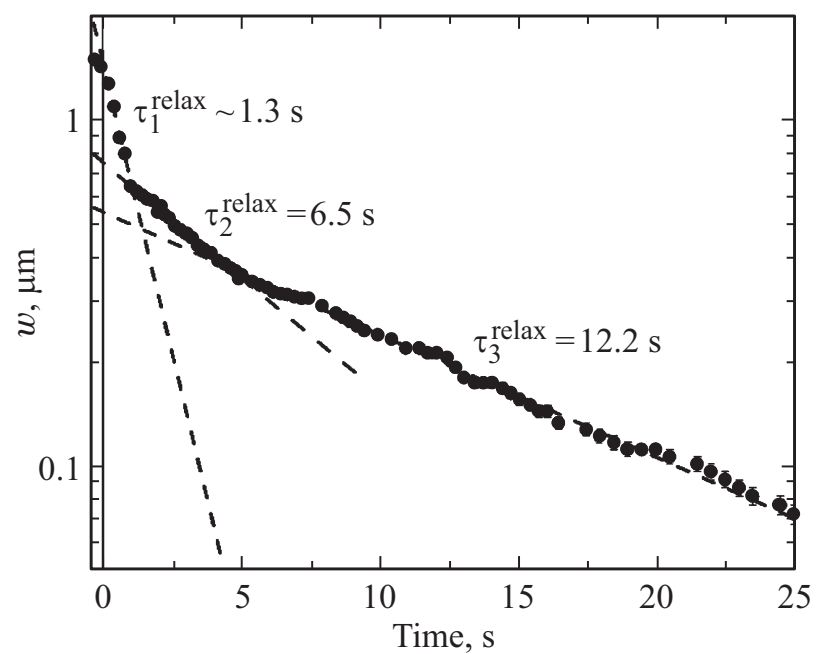

Рис. 2. Релаксация деформации изгиба (прогиб) пластины $\mathrm{SrTiO}_{3}$, после снятия нагрузки $0.5 \mathrm{~N}$. Тонкими пунктирными линиями показан результат соответствия формулы (5) с экспериментом.

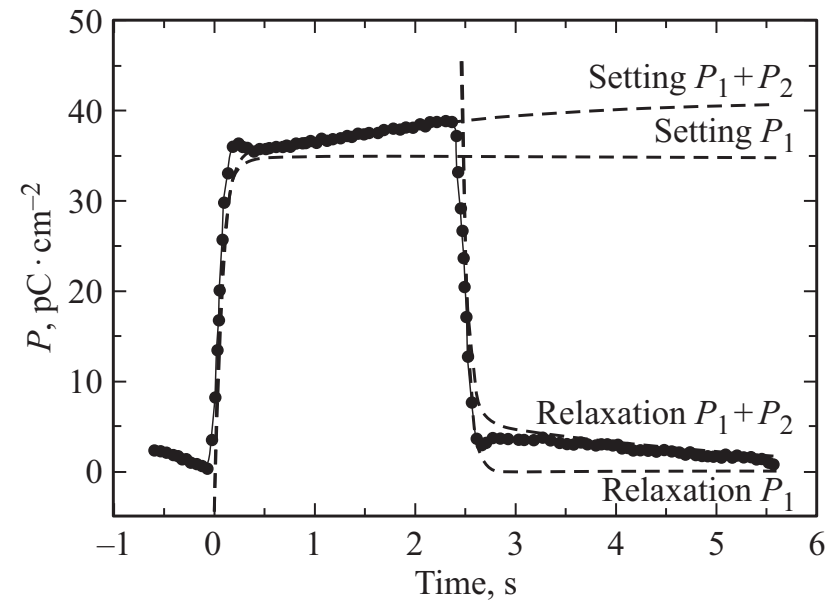

Рис. 3. Импульс поляризационного отклика на механический импульс с нагрузкой $0.5 \mathrm{~N}$. Тонкими пунктирными линиями показано соответствие формул (6) и (7) с экспериментом.

\section{2. Поляризация, индуцированная сферическим изгибом и процессы ее установления и релаксации}

На втором этапе производилось измерение зависимости наведенной поляризации от величины деформации. Импульс механической нагрузки, временная зависимость которого имеет прямоугольную форму, индуцировал импульс поляризации, изображенный на рис. 3. В момент включения механической нагрузки наблюдается процесс установления поляризации, состоящий из быстрого и более медленного возрастания величины поляризации. Зависимость поляризации от времени можно описать следующим экспоненциальным выражением:

$$
P=P_{01}\left[1-\exp \left(-t / \tau_{1}^{\mathrm{set}}\right)\right]+P_{02}\left[1-\exp \left(-t / \tau_{2}^{\mathrm{set}}\right)\right],
$$

где $P_{01}$ и $P_{02}-$ величины вкладов от быстрого и медленного процессов установления поляризации, $\tau_{1}^{\text {set }}$ и $\tau_{2}^{\text {set }}$ - соответствующие им характерные времена. Выключение механической нагрузки сопровождается процессом релаксации поляризации, который также состоит из быстрого и более медленного уменьшения величины поляризации. Временная зависимость процесса релаксации также может быть описана как:

$$
P=P_{01} \exp \left(-t / \tau_{1}^{\text {relax }}\right)+P_{02} \exp \left(-t / \tau_{2}^{\text {relax }}\right),
$$

где $\tau_{1}^{\text {relax }}$ и $\tau_{2}^{\text {relax }}-$ характерные времена релаксации быстрого и медленного процессов.

Из рис. 3 видно, что уравнения (6) и (7) в хорошем согласии с экспериментальной временной зависимостью поляризации $P(t)$, за исключением небольших всплесков в момент окончания быстрых процессов и начала медленных процессов установления/релаксации. Эти всплески связаны с динамическим отскоком пластин, характерной реакцией упругих твердых тел на короткие по времени импульсы механических нагрузок. 

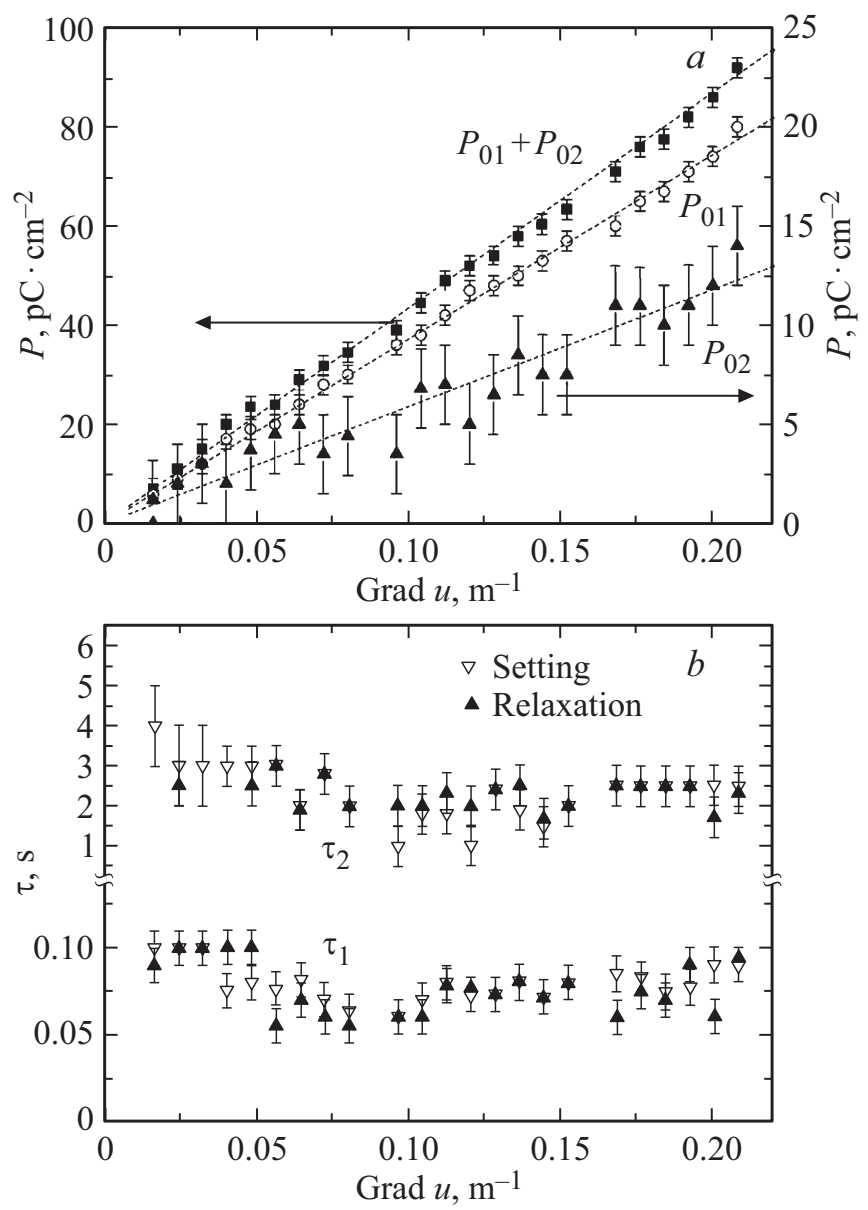

Рис. 4. Зависимости от градиента деформации $а$ ) поляризации и $b$ ) характерных времен установления и релаксации поляризации.

На рис. 4 представлены отдельные зависимости вкладов $P_{01}, P_{02}$ и их суммы $P$ от градиента деформации $\operatorname{grad} u$, который, в свою очередь, получен из величины прогиба (см. [15]). Из рисунка 4 видно, что зависимости $P(\operatorname{grad} u)$ и $P_{01}(\operatorname{grad} u)$ и $P_{02}(\operatorname{grad} u)$ являются линейными. По коэффициентам наклона легко найти эффективные флексоэлектрические коэффициенты: $\widetilde{\mu}_{12}=4.3$, $\widetilde{\mu}_{12}^{01}=3.7$ и $\widetilde{\mu}_{12}^{02}=0.6 \mu \mathrm{C} / \mathrm{m}$, соответственно. Характерные времена установления и релаксации примерно одинаковы и оцениваются величинами $\tau_{1}^{\text {set/relax }}=0.06-0.1 \mathrm{~s}$ и $\tau_{2}^{\text {set/relax }}=1-4 \mathrm{~s}$ для быстрого и медленного процесca, соответственно. Эти величины мало зависят от величины деформации, однако, возможно, существует тенденция к их росту при малых деформациях.

\section{3. Зависимость поляризации от температуры}

Температурные зависимости основных параметров импульса полярного отклика $P_{01}, P_{02}, \tau_{1}^{\text {set }}, \tau_{2}^{\text {set }}$ и $\tau_{1}^{\text {relax }}$, $\tau_{2}^{\text {relax }}$ на импульс нагрузки амплитудой $0.5 \mathrm{~N}$ представлены на рис. 5. Поляризация $P_{01}$ для быстрого процесса монотонно возрастает при понижении температуры от
300 до $200 \mathrm{~K}$ (рис. 5, a). На врезке этого рисунка видно, что зависимость обратной величины этой поляризации хорошо описывается выражением $1 / P_{01}=\left(T-T_{C f}\right) / C_{f}$ со следующими параметрами: $C_{f}=9 \cdot 10^{-5} \mathrm{~K} \cdot \mathrm{m}^{2} / \mathrm{C}$ и $T_{C f}=74 \mathrm{~K}$. В случае медленного процесса, для величины $P_{02}$ наблюдается немонотонный рост с понижением температуры (рис. 5, b). Ниже $200 \mathrm{~K}$ оба процесса сопровождаются резким возрастанием величин $P_{01}$ и $P_{02}$ и заметным разбросом от импульса к импульсу.

На рис. 5, $c$ и $d$ показаны температурные зависимости характерных времен установления и релаксации поляризации. Для быстрого процесса времена установления и релаксации поляризации, $\tau_{1}^{\text {set }}$ и $\tau_{1}^{\text {relax }}$ возрастают с уменьшением температуры и, в рамках погрешности измерений, хорошо описываются соотношением Аррениуса $\tau_{1}=\tau_{01} \exp \left(E_{a} / k T\right)$ (рис. 5, $c$ врезка),
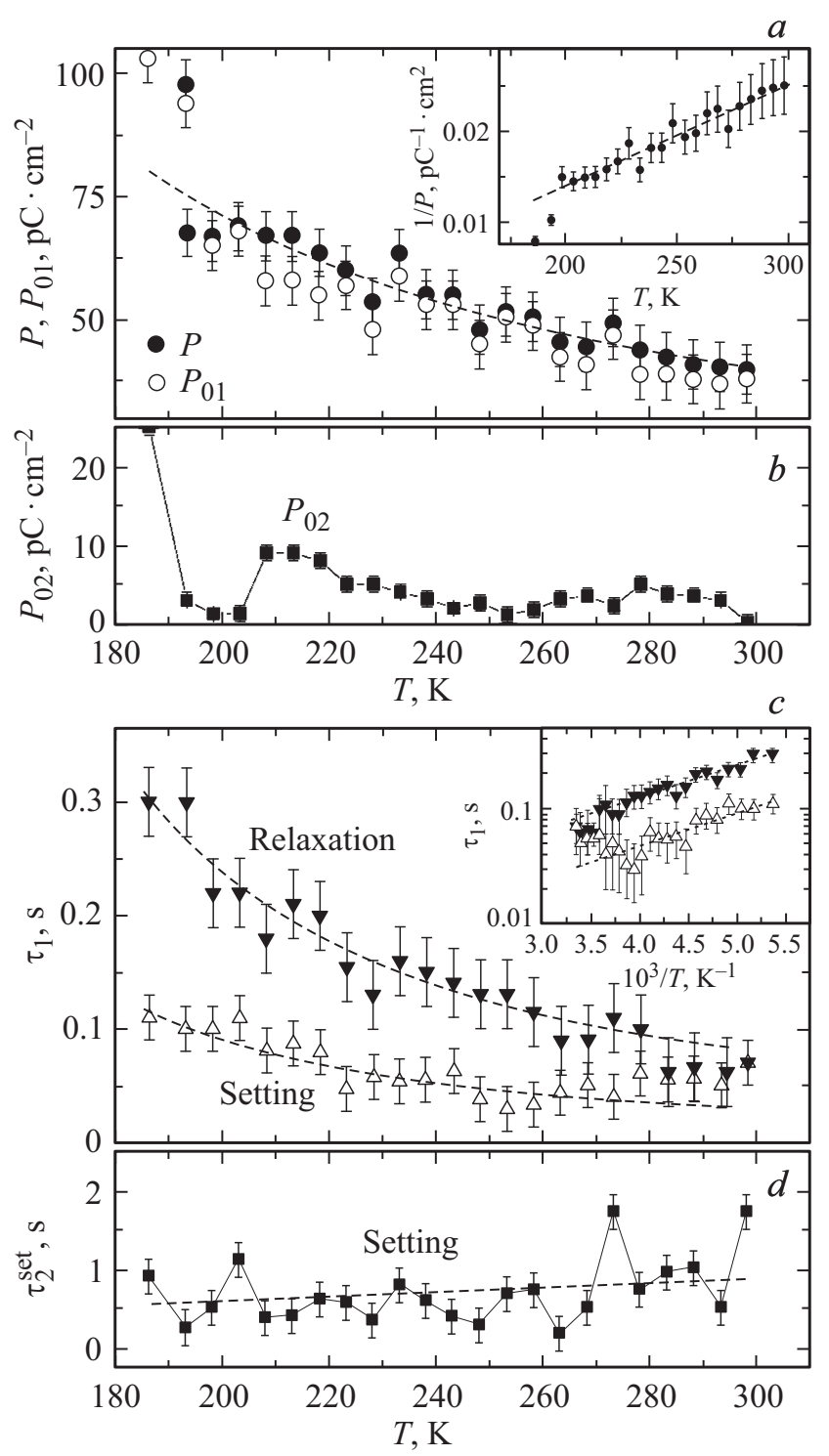

Рис. 5. Температурная зависимость $a, b)$ индуцированной поляризации и $c, d$ ) характерных времен процессов установления/релаксации поляризации. 
где $\tau_{01}-$ константа, $E_{a}-$ энергия активации, $k-$ постоянная Больцмана. Для процесса установления поляризации получены следующие параметры: $E_{a}=650 \mathrm{~K}$ $(56 \mathrm{meV})$ и $\tau_{01}^{\mathrm{set}}=3.5 \cdot 10^{-3} \mathrm{~s}$, а для процесса релаксации: $E_{a}=650 \mathrm{~K}(56 \mathrm{meV}), \tau_{01}^{\text {relax }}=9.2 \cdot 10^{-3} \mathrm{~s}$. Что касается медленного процесса поляризации, то характерное время $\tau_{2}(T)$ варьируется около величины $1 \mathrm{~s} \mathrm{c} \mathrm{разбросом} \mathrm{от}$ 0.5 до $1.5 \mathrm{~s}$ и мало зависит от температуры, однако, возможно, имеется тенденция к возрастанию этой величины в области высоких температур (рис. $5, d$ ).

\section{4. Обсуждение результатов}

Эффективный поперечный флексоэлектрический тензор $\widetilde{\mu}_{12}=4.3 \mu \mathrm{C} / \mathrm{m}$ совпадает с величинами $\widetilde{\mu}_{12}^{+}=4.6$ и $\widetilde{\mu}_{12}^{-}=3.7 \mu \mathrm{C} / \mathrm{m}$, которые были получены при исследовании обратного флексоэлектрического эффекта $[7,14]$. Из эффективного поперечного тензора $\widetilde{\mu}_{12}$ можно вычислить величины продольного $\mu_{11}$ и поперечного $\mu_{12}$ тензоров (см. [4-6]), связанных между собой следующим уравнением $\tilde{\mu}_{12}=-v \mu_{11}+(1-v) \mu_{12}[18]$. Оценки дают следующие величины: $\mu_{12}=5.8$ и $\mu_{11}=0.4 \mu \mathrm{C} / \mathrm{m}$. С помощью формулы (3) можно оценить величину размерного фактора: для продольного коэффициента $\lambda=4.6$, а для поперечного - 67. Полученные величины $\lambda$ того же порядка, что и для титаната бария и соединений на его основе $[10,11]$ и свидетельствуют в пользу применимости эмпирического соотношения (3).

Величина флексоэлектрического эффекта, согласно (3) и ранее опубликованным данным [5,7], должна коррелировать с диэлектрической проницаемостью, которая, в свою очередь, в интервале температур выше $110 \mathrm{~K}$ следует закону Кюри-Вейсса. Зависимость $P_{01}(T)$ для быстрого процесса установления/релаксации поляризации на рис. 5, $а$ действительно следует этому закону в интервале температур 200-300 К. Более того, параметр $T_{C f}=74 \mathrm{~K}$ почти совпадает с параметром $T_{C f}=70 \mathrm{~K}$, который был получен для случая обратного флексоэлектрического эффекта в интервале температур $120-300 \mathrm{~K}$ [7]. Из этого следует, что поляризация $P_{01}$ с более коротким временем установления/релаксации имеет флексоэлектрическое происхождение. Ниже $200 \mathrm{~K}$ резкое возрастанием величин $P_{01}$ и $P_{02}$ и их заметный разброс можно отнести к началу процесса образования доменов (см. [5]) вследствие неоднородного механического напряжения и резкого его изменения в момент контакта механического зонда с поверхностью кристалла.

Полученные тензоры на три порядка больше величин $\mu_{11}=-0.26 \mathrm{nC} / \mathrm{m}$ и $\mu_{12}=-3.74$, теоретически рассчитанных в [4] и экспериментально измеренных $\mu_{11}=0.2$ и $\mu_{12}=7 \mathrm{nC} / \mathrm{m}$ по данным прямого [5] и $\widetilde{\mu}_{12} \approx 4.6 \mathrm{nC} / \mathrm{m}$ обратного метода [6]. В перечисленных экспериментах использовалась переменная по времени механическая нагрузка частотой $30-40 \mathrm{~Hz}$, а во втором эксперименте переменное электрическое поле частотой $10 \mathrm{kHz}$.
При таких частотах многие релаксационные механизмы оказываются выключенными, например, механическая релаксация с временным параметром $\tau_{w 1}^{\text {relax }}=1.3 \mathrm{~s}$ и другие процессы, к которым можно отнести медленные релаксационные вклады в диэлектрическую восприимчивость ионного и электронного происхождения. Более того, в работах $[19,20]$ уже сообщалось о значительном усилении флексоэлектрического эффекта в кристаллах за счет большой концентрации кислородных вакансий и за счет увеличения числа фотоиндуцированных носителей заряда. Поэтому можно говорить о том, что релаксация флексоэлектрического отклика может быть связана с транспортом и перераспределением носителей заряда (электронов, ионов и вакансий) [12].

Рассмотрим наиболее вероятные механизмы влияния носителей заряда и дефектов кристаллической решетки на флексоэлектрический эффект. С одной стороны, перераспределение заряда можно отнести к действию химического давления вследствие неоднородного искажения кристаллической решетки при неоднородном механическом напряжении (эффект Вегарда) [12]. Другой причиной может быть дрейф носителей заряда: электронов, ионов и заряженных вакансий во внутреннем поле флексоэлектрического происхождения $E_{\mathrm{Flex}}$. Известно, что кислородные вакансии отвечают за пластичность кристалла ST. В нашем случае, релаксация механического напряжения за счет пластичности кристалла характеризуется временами 1.3, 6.5 и $12.5 \mathrm{~s}$ (рис. 2). По-видимому, релаксация, обусловленная пластичностью, также определяет и время $\tau_{2}=1-4 \mathrm{~s}$ для медленной релаксации поляризации $P_{02}$. Это характерное время мало зависит от температуры, что может быть связано с тем, что энергия термоактивации миграции кислородных вакансий оценивается величиной порядка $0.6-0.7 \mathrm{eV}[21]$, а энтальпия для активации дислокаций - величиной 2-6eV [22]. Что касается быстрого процесса установления/релаксации поляризации, то его характерное время $\tau_{1}$ на порядок меньше релаксации механического напряжения и имеет термоактивационный характер с энергией $56 \mathrm{meV}$. По данным работ [23,24] в ST такая величина энергии порядка $10-70 \mathrm{meV}$ характерна для ионизации кислородных вакансий по схеме $V_{\mathrm{O}}^{\bullet \bullet}+2 \mathrm{e}^{-}$. В таком случае, релаксация поляризации $P_{01}$ обусловлена движением электронов во внутреннем поле $E_{\text {Flex }}$ и захватом электронов ловушками, связанных с вакансиями кислорода.

Перераспределение электронов и их взаимодействие с центрами $V_{\mathrm{O}}$ в объеме кристалла также может объяснить небольшую биполярность в величинах $\widetilde{\mu}_{12}^{+}=4.6$ и $\widetilde{\mu}_{12}^{-}=3.7 \mu \mathrm{C} / \mathrm{m}$, которая наблюдалась при обратном флексоэлектрическом эффекте [7,14]. Эта биполярность проявляется в том, что около положительно заряженного электрода поверхность испытывает большую деформацию, чем около электрода отрицательной полярности. Из [25] известно, что дважды ионизованная кислородная вакансия $V_{\mathrm{O}}^{\bullet \bullet}$ увеличивает расстояние между соседними катионами до 4\% в силу кулоновского отталкивания. 
С другой стороны, электроны, захваченные вакансией, должны уменьшать это отталкивание, вызывая сокращение этого расстояния. Отсюда следует, что приповерхностные области с повышенной концентрацией электронов около положительного электрода должны вызывать больший изгиб поверхности, чем поверхность около отрицательного электрода с вогнутой стороной, что и наблюдалось в ряде экспериментов [14,20].

\section{5. Выводы}

Проведено исследование статического прямого флексоэлектрического эффекта, при котором измерялась величина поляризации, индуцированной сферическим изгибом тонких пластин монокристаллов $\mathrm{SrTiO}_{3}$.

Измерен эффективный поперечный флексоэлектрический тензор $\widetilde{\mu}_{12}=4.3 \mu \mathrm{C} / \mathrm{m}$, из которого определены продольный и поперечный тензоры $\mu_{11}=0.4$ и $\mu_{12}=5.8 \mu \mathrm{C} / \mathrm{m}$.

Исследованы процессы релаксации неоднородной деформации и индуцированной поляризации и получены характерные времена этих процессов. Приведены результаты сравнения прямого и обратного эффекта.

\section{Благодарности}

Авторы благодарят В.А. Трепакова за полезную консультацию.

\section{Конфликт интересов}

Авторы заявляют, что у них нет конфликта интересов.

\section{Список литературы}

[1] A.K. Tagantsev, P.V. Yudin. Flexoelectricity in solids. World Scientific Publishing Co., Singapore (2017). 396 p.

[2] P. Zubko, G. Catalan, A. Tagantsev. Ann. Rev. Mater. Res. 43, 387 (2013).

[3] B. Wang, Y. Gua, Sh. Zhang, L.-Q. Chen. Prog. Mater. Sci. 106, 100570 (2019).

[4] R. Maranganti, P. Sharma. Phys. Rev. B 80, 054109 (2009).

[5] P. Zubko, G. Catalan, A. Buckley, P. Welche, J. Scott. Phys. Rev. Lett. 99, 167601 (2007).

[6] U.K. Bhaskar, N. Banerjee, A. Abdollahi, Z. Wang, D.G. Schlom, G. Rijnders, G. Catalan. Nature Nanotechnology 11, 263 (2016).

[7] В.Г. Залесский, Е.Д. Румянцева. ФТТ 56, 7, 1301 (2014).

[8] Ch. Wang, Sh. Wang, W. Huang, H. Xie, L. Cai, F.M. Zhang, X.S. Wu. Appl. Phys. Lett. 113, 242903 (2018).

[9] А.К. Таганцев. ЖЭТФ 88, 2108 (1985).

[10] W.H. Ma, L.E. Cross. Appl. Phys. Lett. 81, 3440 (2002).

[11] W.H. Ma, L.E. Cross. Appl. Phys. Lett. 88, 232902 (2006).

[12] A.N. Morozovska, E.A. Eliseev, G.S. Svechnikov, S.V. Kalinin. Phys. Rev. B 84, 045402 (2011).

[13] A. Nakamura, K. Yasufuku, Y. Furushima, K. Toyoura, K. Peter D. Lagerlöf, K. Matsunaga. Crystals 7, 351 (2017).
[14] Е.Д. Обозова, В.Г. Залесский, Письма в ЖЭТФ 103, 11, 792 (2016).

[15] Е.Д. Obozova, П.П. Сырников, В.Г.Залесский. Приборы и техника эксперимента 6, 90 (2019).

[16] S. Timoshenko, S. Woinowsky-Krieger. Theory of plates and shells, Mc Graw-Hill Co, N. Y. (1959), 580 p.

[17] R. Gaillac, P. Pullumbi, F.X. Coudert. J. Phys.: Condens. Matter. 28, 275201 (2016).

[18] Э.В. Бурсиан, Н.Н. Трунов. ФТТ 16, 1187 (1974).

[19] J. Narvaez, F. Vasquez-Sancho, G. Catalan. Nature 538, 219 (2016).

[20] Y. Jin, F. Zhang, K. Zhou, Ch.H. Suen, X.Y. Zhou, J.-Y. Dai, Appl. Phys. Lett. 118, 164101 (2021).

[21] R.A. De Souza. Adv. Funct. Mater. 25, 6326 (2015).

[22] S. Taeri, D. Brunner, W. Sigle, M. Rühle. Z. Metallkd. 95, 433 (2004).

[23] V.V. Laguta, M.D. Glinchuk, I.P. Bykov, A. Cremona, P. Galinetto, E. Giulotto, L. Jastrabik, J. Rosa. J. Appl. Phys. 93, 6056 (2003).

[24] В.А. Трепаков, В.С. Вихнин, П.П. Сырников, Ф. Смутный, М. Савинов, Л. Ястрабик. ФТТ 39, 11, 2040, (1997).

[25] T. Hoshina, R. Sase, J. Nishiyama, H. Takeda, T. Tsurumi. J. Ceram. Soc. Jpn 126 [5], 263 (2018).

Редактор Д.В. Жуманов 